\title{
Gut content metabarcoding reveals potential importance of fisheries discards consumption in marine fauna
}

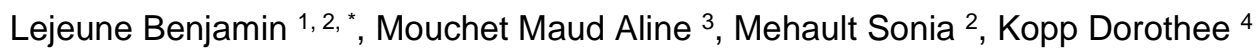

${ }^{1}$ Museum National d'Histoire Naturelle, 52827, Paris, Île-de-France, France

2 Ifremer, Station de Lorient, 121246, Lorient, Bretagne, France;

${ }^{3}$ Museum National d'Histoire Naturelle, 52827, Paris, Île-de-France, France;

4 Ifremer, Station de Lorient, 121246, Laboratoire Technologie et Biologie Halieutique, Lorient, Bretagne, France;

* Corresponding author : Benjamin Lejeune, email address : Benjamin.Lejeune@mnhn.fr

\begin{abstract}
:
Fisheries discards have become a source of concern for the perennation of marine resources. To reduce discards, the European Union adopted a Landing Obligation under the reform of its Common Fisheries Policy. However, food web consequences of reducing discards remain uncertain since their degree and pathway of reintegration are understudied. We used multi-marker DNA metabarcoding of gut contents and an ecological network approach to quantify marine fauna reliance on discarded fish and functional importance of discard consumers in coastal fishing grounds. We show that potential discard consumption is widespread across fish and invertebrates, but particularly important for decapods which were also pinpointed as functionally important. Potential discard consumption may represent up to $66 \%$ of all interactions involving fish prey in the reconstructed network. We highlight that discard reliance may be more important than previously assessed in some fishing areas and support functionally important taxa. While reducing discarding remains a conservation priority, it is crucial to understand discards reintegration in marine food webs to anticipate changes in the context of an ecosystem approach to fisheries management.
\end{abstract}

Keywords : Diet, Discard ban, DNA metabarcoding, Fishing impact, Landing Obligation, Marine food web, Molecular network analysis, Scavengers, Trophic ecology 


\section{Introduction}

35 Fishing activity is considered one of the most important threats to marine ecosystems

36 worldwide (Jackson et al. 2001; Guillen et al. 2018). The impact of fishing activity on marine

37 ecosystems has been mainly studied with respect to the removal of species from their

38 environment, and its direct and indirect effects, which can have complex cascading effects on

39 food web structure and stability, ultimately affecting ecosystem functioning (Bascompte et al., 
2005; Scheffer et al., 2005). Yet, another aspect of fisheries activities that could significantly impact food web structure and ecosystem functioning is fisheries discards (i.e. animals caught, but returned to the sea, dead or alive). Discarding arises from economic or legislative imperatives (low commercial value, over quota restriction, below the minimum allowable size, damaged catches; Kelleher, 2005; Zeller et al., 2018). Discards are estimated to represent between 7 and 10 million tons per year at a global scale, representing almost $10 \%$ of global catches (Kelleher 2005; Zeller et al. 2018). In Europe, the North-East Atlantic ocean has been identified as a 'discard hotspot' (Guillen et al. 2018), with fishing activities that can reach up to $50 \%$ discard rates, as in the case of Nephrops fishery in the Bay of Biscay (Kelleher 2005). Because of the considerable discard rates of some fishing practices, they are now also being recognized as an important factor of biomass dispersion and have become a cause of concern for the perennation of marine resources in many countries (Zeller et al. 2018). The European Union adopted a discard ban in the form of a Landing Obligation (LO) under the reform of its Common Fisheries Policy, with a gradual implementation over the years 2015-2019 (European Union 2013). This reform applies a restriction on the discarding of commercial species under total allowable catches (TAC) and quota, have a minimum landing size, as well as an obligation to return prohibited species to the sea. However, the short- and long-term environmental effects of reducing fishing discards remain uncertain since their degree and pathway of reintegration into the marine food web are understudied, albeit being topics of growing interest (Jenkins et al., 2004; Kopp et al., 2016; Sherley et al., 2019). Understanding the importance of fisheries discards reintegration in marine food webs is crucial in the context of an ecosystem approach to fisheries management, both to understand how fishing affects the functioning of marine ecosystems and to predict the impact of a reduction in fisheries discards on marine communities. 
Scavenging behaviors and interactions with fisheries have been well documented in seabirds worldwide (Karris et al., 2018; Votier et al., 2013), with evidence that discards may support a large community of scavenger seabirds and be an important determinant of population dynamics (Sherley et al. 2019). However, a substantial part of discards is not directly consumed by seabirds (Garthe et al., 1996), and sinks through the water column, ending up on the seabed (Hill \& Wassenberg 2000). Multiple studies have demonstrated that some marine species have developed scavenging feeding strategies targeting dead or damaged fauna originating from fishing activities, including fisheries discards (Groenewold \& Fonds, 2000; Jenkins et al., 2004; Ramsay et al., 1997). Results from these studies suggest that constant input of organic matter in the form of fisheries discards might play an important role in shaping marine communities and food webs. Such scavenging behaviors could potentially impact marine food webs in diverse ways, by resupplying a part of the biomass extracted by fisheries to the scavenger community and potentially other non-typically scavenger species, and enhancing secondary production (Bluhm \& Bechtel 2003). It could also disproportionately affect some species compared to others depending on their level of specialization on fisheries discards, as is suspected in seabirds (Tyson et al., 2015). Yet, to what extent such scavenging behaviors may be common and determine the diet of marine fauna, including that of non-typical scavengers (Olaso et al., 2002) remains largely unknown. These questions are timely since depending on the existence and strength of trophic links with fisheries discards, current efforts to limit discarding practices, including the LO, may directly affect species feeding in fishing grounds, community stability and potentially propagate changes through the marine food web (Kopp et al. 2016).

Estimation of discard consumption by marine fauna is hindered by inherent limitations of traditionally used diet assessment techniques. On the one hand, studies on discard consumption have relied mainly on baited remote underwater video systems (e.g. Bluhm \& 
89 Bechtel, 2003; Hill \& Wassenberg, 2000; Ramsay et al., 1997), yielding results that do not 90 allow one to ponder the importance of fisheries discards consumption relative to other items in 91 the diet at a population level. On the other hand, traditional gut content and stable isotope analyses which constitute the main techniques used to assess discard contribution to the diet of marine fauna often only allow broad trophic assignments of consumers. DNA metabarcoding useful to address some of the shortfalls of these techniques to study the fate of fisheries discards (Mcinnes et al. 2017). It is a well-established technique that can yield unparalleled resolution in taxonomic identification and is being increasingly used to document trophic interactions in marine ecosystems (Casey et al. 2019; Takahashi et al. 2020). Finally, discards identity and rates may vary considerably across time and geographic location, making it impossible to generally classify some prey as being always ingested as discards and others as originating from 'natural' predation (Zeller et al. 2018). Coupling knowledge of local discard identity and rates with prior knowledge of predator-prey interaction of the studied consumers may allow to classify ingested prey according to their probability of originating from fisheries discards, providing that the time frame of discard frequencies and the trophic tracer are similar.

In this study, we first aim to quantify the potential consumption of fish originating from

107 fisheries discards in marine fauna feeding in coastal fishing grounds, by using multi-marker 108 DNA metabarcoding of gut contents. We developed an original approach to evaluate the 109 probability of prey items as originating from fisheries discards based on prior knowledge of 110 predator-prey interactions, consumers trophic guilds and a record of local discarding activity 111 based on data collected during the study period, the whole year and the year before. Second, 112 we aim to identify whether discarded taxa or taxa relying on fisheries discards may constitute 113 functionally important nodes within the local network of trophic interactions by using a 
114 molecular ecological network approach and therefore identify the potential pathways through 115 which the LO may propagate changes through the food web. We hypothesized that (1) all 116 sampled consumers within the fishing ground community would have ingested fish likely 117 originating from discarding practices (i.e. even non-typically scavenging, non-piscivorous and

118 low trophic level species), but that this resource would be more important for some species than 119 others and particularly for benthic invertebrate macrofauna; and (2) potential discard 120 consumption would represent a significant portion of all trophic interactions within the 121 reconstructed network and support functionally important taxa such as decapods, which may 122 therefore trigger cascading effects potentially affecting food web and community structure 123 following discards reduction.

\section{Material and methods}

\section{Gut content and local discarding data collection}

127 Data were collected in April 2019 in the Bay of Bourgneuf (Bay of Biscay, France, northeast 128 Atlantic). Despite being shallow (from 0 to $34 \mathrm{~m}$ depth) and covering a relatively small area $129\left(320 \mathrm{~km}^{2}\right)$, this bay is diverse, both in terms of substrate type (i.e. composed of a variety of 130 patchy rocky, sandy and muddy bottoms) and species occurrence (Gauduchon et al. 2020). Both 131 sampling for gut content analysis and recording of local discarding activity at the time of 132 sampling (identity and importance of discards) were carried out onboard of the same fishing 133 boat, a $10.95 \mathrm{~m}$ long commercial trawler rigged with a single bottom trawl used to target 134 multispecies fish assemblages (20 m headline and $70 \mathrm{~mm}$ diamond mesh codend) and are based 135 on 12 trawl hauls over a 3 days period during normal operation of this boat. This trawler is 136 representative of the commercial trawling fleet of the Bay of Bourgneuf, which are $<12 \mathrm{~m}$ long 137 (Ifremer. Système d'Informations Halieutiques 2020). Among fishing activities in the ICES 8a 138 and $\mathrm{b}$ divisions where our sampling took place, bottom trawling is the main source of discards 
139 (i.e. 5000 t/year; Kopp et al., 2016). Coupling sampling and discarding observation was done 140 to obtain information on the identity and importance of discarded species at a timescale as close 141 as possible to that of diet information provided by gut content metabarcoding and at the exact 142 same location. Our own in situ observations of discarding at the time of sampling were further 143 compared and complemented using data from the OBSMER database (Cornou et al. 2021). 144 OBSMER is a sampling program that separately collects catch and discard data (species 145 identity, tonnage and sizes) of commercial fisheries on all French maritime facades. Data are 146 collected by at-sea observers on board of professional fishing vessels. They monitor a complete 147 fishing trip and randomly sample one-third to half of fishing operations of the vessel during 148 each trip. Data request from OBSMER concerned years 2018 and 2019, métier OTB_DEF and 149 OTB_CEP, and ICES sub-division 23E7BB. These data were used to calculate a 'discard 150 importance score' for each prey item as follows: $0=$ No discarding observed in any dataset, 1 151 = low discard importance (i.e. species was discarded and met the following criteria: (a) 152 discarded in less than $25 \%$ of the hauls and with less than 10 individuals per haul according to 153 our own observational data OR (b) discarded in mean quantities (weight) per haul lower than 154 the mean of all discarded fish per haul according to OBSMER data) and $2=$ high discard 155 frequency (all discarded species above the aforementioned thresholds in at least one dataset; 156 see Table S1 for details).

157 Individuals captured for gut content analysis were directly frozen to be further dissected in 158 the lab, except for Conger conger, Raja undulata and Scyliorhinus canicula for which only the 159 digestive tract was frozen due to their large body size (dissected on board). In total, 160 metabarcoding was performed on 369 individuals belonging to 22 consumer taxa spanning 7 161 different Classes which are representative of marine fauna and size classes captured by commercial trawling in the bay. All data were collected and analyzed in accordance with the 
163 authorizations and ethics approval delivered by the Ministère de la transition écologique et

164 solidaire (NOR: TREL1902817S / 168).

\section{Gut content acquisition}

167 Dissections were conducted in sterile conditions (i.e. equipment and surfaces were disinfected 168 and sterilized between dissections by bleaching and flame sterilization). Frozen individuals 169 were thawed at ambient temperature and subsequently dissected to extract the digestive tract. 170 Digestive content was scraped along the entire length of the digestive tract, homogenized and 171 finally sub-sampled to a maximum of $15 \mathrm{~g}$ that was preserved dried in silica gel.

\section{Production of metabarcoding data}

174 Samples preserved in silica gel were sent to Argaly SA (Sainte-Hélène-du-Lac, France; 175 https://www.argaly.com) for metabarcoding. Each sample was ground and homogenized using 176 a mortar. DNA extractions were carried out using the NucleoSpin Soil kit (Macherey-Nagel, 177 Düren, Germany) according to the manufacturer's instructions. The final elution was performed with $100 \mu \mathrm{L}$ of SE buffer and diluted 5 times prior to PCR (final volume $=0.5 \mathrm{~mL}$ ). A combination of two complementary primer pairs was used for diet assessment: 'Euka02' (TTTGTCTGSTTAATTSCG and CACAGACCTGTTATTGC; Guardiola et al., 2015),

181 targeting the 18S rDNA region was used as a generalist marker to obtain a full picture of the 182 animal fraction of the diet, and 'Tele02' (AAACTCGTGCCAGCCACC and 183 GGGTATCTAATCCCAGTTTG; Taberlet et al., 2018) targeting the 12S mitochondrial rDNA 184 was used to characterize more precisely the ingested fish (Actinopterygii and Chondrichthyes). 185 Together, they allow to globally estimate the fraction of the ingested fish within the animal 186 fraction of the diet of each consumer. Amplification of each DNA extract was carried out in 187 four independent PCR replicates, for each marker. Bioinformatic discrimination of PCR 
188 replicates after sequencing was allowed by adding tags to the 5 ' end of both the forward and 189 reverse primers so that each PCR replicate was represented by a unique combination of forward 190 and reverse tags. Tags were constituted of an eight-nucleotide sequence, with at least five 191 nucleotide differences among them (Coissac 2012) to which two to four random nucleotides 192 were added (on the 5' end), to ensure sufficient sequence diversity and efficient detection of 193 clusters during sequencing. PCR amplifications were performed in a $20-\mu \mathrm{L}$ volume containing $1942 \mu \mathrm{L}$ of DNA extract, $0.4 \mu \mathrm{M}$ of each primer, $10 \mu \mathrm{L}$ of AmpliTaq Gold 360 Master Mix 195 (Applied Biosystems, Foster City, CA, USA) and $0.16 \mu \mathrm{L}$ of $20 \mathrm{mg}^{-\mathrm{ml}^{-1}}$ bovine serum albumin 196 (BSA; Roche Diagnostics, Basel, Switzerland). Thermocycling conditions included an initial 197 DNA polymerase activation step of $10 \mathrm{~min}$ at $95^{\circ} \mathrm{C}, 45$ cycles with a 30 s denaturation at $95^{\circ} \mathrm{C}$, 198 a $30 \mathrm{~s}$ annealing at $45^{\circ} \mathrm{C}$ for Euka02 and $54^{\circ} \mathrm{C}$ for Tele 02 , and a 60 s elongation at $72^{\circ} \mathrm{C}$, followed 199 by a final extension step of $7 \mathrm{~min}$ at $72^{\circ} \mathrm{C}$. Purification of PCR products (combined in equal 200 volumes), were conducted using the MinElute PCR purification kit (Qiagen, Hilden, Germany) 201 following the manufacturer's instructions. Gel electrophoresis (E-Gel Power Snap; Invitrogen, 202 Carlsbad, CA, USA) was used to check for amplification and purification success. Library 203 preparation and sequencing were outsourced to Fasteris SA (Geneva, Switzerland; 204 https://www.fasteris.com/dna/). Two sequencing libraries (one per marker) were prepared 205 following the PCR-free MetaFast protocol (Taberlet et al. 2018), which limits the formation of 206 chimeras. All amplicons were sequenced on a NextSeq 500 sequencing platform (Illumina, San 207 Diego, CA, USA), using the rapid run mode generating 250 bp paired-end sequences. 208 Extraction and PCR negatives (20 at each step, i.e. one by extraction batch and PCR plate), as 209 well as unused tag combinations ( 240 in total, i.e. 12 by PCR plate) were included to control 210 for potential contaminations and false positives caused by tag jumps (Schnell et al., 2015). 
213 The OBITools package (Boyer et al. 2015) was used to analyse raw reads. First, paired-end 214 reads were assembled with illuminapairedend function, only retaining sequences with an 215 alignment score higher than 40. Second, ngsfilter function was used to assign aligned sequences 216 to the corresponding sample by allowing two and zero mismatches on primers and tags, 217 respectively. Third, obiuniq function was used for sequence dereplication. Sequences whose 218 length fell outside the expected size interval (shorter than $36 \mathrm{bp}$ for Euka02, or $129 \mathrm{bp}$ for 219 Tele02), bad-quality sequences (i.e. containing " $N$ ") and those never observed at least ten times 220 in at least one PCR replicate were filtered out. Finally, ecotag function was used to perform 221 taxonomic assignment of each molecular Operational Taxonomic Unit (mOTU), for each 222 marker, by comparing the mOTU sequence to a database of full-length reference metabarcodes. 223 This reference database was constructed combining local reference sequences (Table S2), and 224 sequences obtained from the GenBank public database (release 135) using ecoPCR (Ficetola et 225 al., 2010). More specifically, ecoPCR carried out an in silico PCR on GenBank with the primer 226 pair used for the experiment and allowing three mismatches per primer. The obtained reference 227 sequences were further curated by keeping only sequences assigned at least at the family level. 228 For Euka02, when a mOTU was $100 \%$ identical with a sequence from the local reference 229 database, this taxonomic assignment was favored in order to avoid any potential dilution of the 230 local taxonomic information with that, probably less relevant, available in GenBank.

232 for further data filtering, removing spurious or contaminant sequences that can bias ecological 233 conclusions drawn from DNA metabarcoding data (Calderón-Sanou et al., 2019). We discarded 234 from our dataset (1) potential chimeras (i.e. mOTUs with a best identity $<95 \%$ with the local 235 reference database or reference sequences from GenBank release 135); (2) contaminants (i.e. 236 mOTUs that were more numerous in the negative control replicates than in true PCR replicates); 237 (3) mOTUs assigned to taxa other than marine organisms for Euka02 and mOTUs assigned to 
238 taxa other than marine fish for Tele02, because Tele02 can also amplify taxa belonging to other 239 classes (mammals, birds); and (4) PCR replicates with a low sequencing coverage. In total, $24040,211,815$ clusters were sequenced for Tele02 and 58,843,304 for Euka02. Total numbers of 241 sequences after data filtering were 15,874,979 and 25,948,409 for Tele02 and Euka02 datasets. 242 After this, we removed 'self-hits' (sequences identified as the studied species within its 243 own gut samples) from both datasets, as these sequences can come from naturally degrading 244 cells from the guts of the studied species and cannot be distinguished from potential 245 consumption of conspecifics, which is considered as an intrinsic limitation of gut content 246 metabarcoding (Taberlet et al. 2018; Casey et al. 2019). Only three species depicted high rates 247 of self-hits in the Euka02 dataset (Aphrodita aculeata, Buccinum undatum and Echinus 248 esculentus), which were the taxa with the lowest sample sizes. High rates of self-hits for a given 249 species were defined as when self-hits were the highest taxonomic assignment across the 250 majority of individuals of that consumer species (Casey et al. 2019), often resulting in self-hit 251 counts being on average higher than total read counts (Table S3). High rates of self-hits were 252 expected in fish species with Tele02 dataset because many individuals which did not consume 253 other fish (e.g. from species not belonging to the piscivore trophic guild; Thompson et al. 2020), 254 would likely only amplify DNA from their own degrading cells with this marker. This was the 255 case for Callionymus lyra, Chelidonichtys lucerna, Conger conger, Pleuronectes platessa and 256 Trisopterus luscus. Total number of sequences after self-hits removal were 9,065,600 and 257 15,191,780 for Tele02 and Euka02, respectively. Count data (number of sequences) were 258 transformed to occurrence data (presence-absence). To avoid rare-item inflation that can bias 259 diet estimation, normalize detection across samples and further limit the risk of potential 260 contaminations to remain in the dataset after data filtering steps, we set a threshold of minimum 261 sequence abundance to determine if a mOTU should be considered as an occurrence. We used 262 a threshold of $\geq 1 \%$ of the total number of sequences amplified in the stomach content to define 
an occurrence (i.e. MOTUs representing $<1 \%$ of the total number of sequences amplified in the stomach of each consumer were removed) (Deagle et al. 2019). Finally, we built a consensus diet incorporating both molecular markers by merging the two datasets (Euka02 and Tele02) into a single one following the multi-marker merging procedure detailed in da Silva et al. (2019). Essentially, this merging procedure assumes that within the same gut content, a given food item recovered at higher taxonomic ranking (e.g. order or family) by the most generalist marker (Euka02) is the same as items of the same taxonomic group recovered at lower taxonomic ranking (e.g. genus or species) by the specialist marker (Tele02). Merged dataset contained a total of 154 identified mOTUs across all samples, with 40 belonging to Actinopterygii and Chondrichthyes.

\section{Calculation of a score of 'probability of consumption as fisheries discard'}

All fish prey were classified according to their probability of being ingested as fisheries discard by calculating a discard probability score (DPS), ranging from 0 to 3 , following: $0=$ very low, $1=$ low, 2 = medium, 3 = high probability of being ingested as fisheries discard (details on DPS calculation for each predator-prey interaction are provided in Table S4). The score was calculated by compiling information on the discarding importance of the prey (i.e. 'discard importance score' detailed above) and known diet and trophic guild affiliation of the consumer (i.e. 'trophic link score'). The 'trophic link score', set either as 0 or 1 was assigned to each consumer-prey combination appearing in the metabarcoding results based on a literature review as follows: $0=$ natural predation is documented in the specific literature of the consumer or possible based on trophic guild affiliation of the consumer (i.e. predation on the family of the prey was observed in other species belonging to the consumer's trophic guild), $1=$ natural predation is not documented either in the specific literature of the consumer or in other species belonging to the consumer's trophic guild. Trophic guild affiliations of fish were derived from 
288 Thompson et al. (2020) which source stomach content records from ICES (1997) and 289 DAPSTOM (Pinnegar 2014) to establish trophic guilds. Specific diet of invertebrate taxa were 290 complemented with the trophic traits 'feeding type', 'feeding habit' and 'trophic level' available 291 from Sealifebase (https://www.sealifebase.ca; Palomares \& Pauly 2021) and the Biological 292 Traits Information Catalogue (www.marlin.ac.uk/biotic; MarLIN 2006). The discard 293 probability score, was calculated in an additive way as DPS = 'discard importance score' + 294 'trophic link score'. Using trophic guilds and traits to determine 'the trophic link score' allows 295 to limit biases and overestimating of discard consumption due to the unequal state of knowledge 296 on the specific diet of the different taxa. Additionally, we adopted a conservative approach to 297 avoid overamplifying the DPS by assigning a DPS $=0$ to all prey items whose 'discard 298 importance score' $=0$. This means that even if predation on a given prey is undocumented to 299 our knowledge and according to the larger spectrum of the trophic guild ('trophic link score' = 300 1), if the prey was not observed being discarded at the time of sampling, the resulting DPS $=0$. 301 Following the same conservative approach, whenever a prey mOTU was identified to the genus 302 level or higher, we assigned the lowest 'discard importance score' of all species included within 303 its taxonomic group.

304

\section{Diet analysis}

306 Information on the occurrence of each mOTU in the gut content of each species was 307 summarized using Frequency of Occurrence (FOO), which is calculated for each consumer 308 species as the number of occurrences of a given mOTU divided by the total number of gut 309 contents samples of that consumer species. The weighted Percentage of Occurrence (wPOO) 310 was obtained following Deagle et al. (2018): occurrences of food items are weighted 311 (standardized) across the entire diet at the individual level, then these weighted occurrences are 
312 summed at the species level and divided by species sample size. Diet richness was assessed at 313 the population and individual levels using raw richness of mOTUs.

\section{Molecular Ecological Network Analysis}

316 We built a network of trophic interactions among consumers sampled in the Bay of Bourgneuf 317 fishing grounds (i.e. species and sizes targeted by commercial trawling) and their prey using 318 gut content metabarcoding data of the 22 consumer species to quantify the importance of 319 trophic links potentially involving discard consumption at the scale of the modelled network, 320 and whether discards may be fueling structurally important species (Meyer et al., 2020). The 321 network was directed from consumers to prey and interactions were weighted using the wPOO 322 values. We used Gephi (version 0.9.2; Bastian et al., 2009) to visualize and calculate all network 323 metrics. First, we mapped the network of trophic interactions using Forced Atlas 2 layout, a 324 force-directed algorithm used to depict the modular aspect of network structure (Jacomy et al., 325 2014). Second, to obtain a view of the importance of trophic links involving discard 326 consumption at the scale of the modeled network, we quantified the proportion of trophic links 327 involving fish prey within the network and among those, the proportion of links likely involving 328 discarded fish (links involving prey with DPS $\geq 2$ ). Third, we measured the relative functional 329 importance of each taxon within the modeled network by calculating: (i) Weighted in-degree 330 centrality (i.e. the number of links directed to the node, weighted according to wPOO values),

331 which indicates the degree to which a taxon is an important food source for other taxa in the 332 network, identifying central taxa whose loss may trigger cascading effects, indirectly impacting 333 the community (Elhesha et al., 2017); (ii) Eigenvector centrality, which measures the node's 334 importance within the network while accounting for the importance of its neighbors, further 335 helping to identify taxa that potentially strongly influence the abundance of others (Allesina \& 336 Pascual 2009). 


\section{$338 \quad$ Results}

\section{Diet composition across the marine community}

340 We sampled 22 consumers for a total of 369 individuals (Table 1). Individual sample size 341 ranged between 4 for Aphrodita aculeata (Polychaeta) and 27 for Sepia officinalis 342 (Cephalopoda), except for Echinus esculentus (Echinoidea) and Buccinum undatum 343 (Gastropoda) which included only one individual. Sample sizes of A. aculeata, E. esculentus 344 and B. undatum were judged too low to allow for any generalization of findings, therefore 345 results concerning these three taxa are presented in tables but not extensively discussed. Total 346 prey richness ranged between 4 and 57 mOTUs for B. undatum and Psammechinus miliaris 347 (Echinoidea), respectively. All 22 consumer taxa ingested fish (i.e. mOTUs identified as 348 Actinopterygii or Chondrichthyes) (Fig. 1; Fig. S1). At the population level, diversity of 349 ingested fish mOTUs ranged between 4 and 24 for $C$. lyra and $S$. officinalis, respectively. It 350 was notably high in the gut contents of $P$. miliaris (18 different fish mOTUs; Table 1), in the 351 two Chondrichthyes Raja undulata and Scyliorhinus canicula (13 and 16 different fish mOTUs, 352 respectively) and in Malacostraca where it ranged between 8 for the spider crab Maja 353 brachydactyla and 19 for the shrimp Crangon sp. In Actinopterygii, diversity of fish prey 354 ranged between 4 for Callionymus lyra and 15 for Trachurus trachurus. At the individual level, 355 richness of prey mOTUs were highest for Crangon sp. S. officinalis and T. trachurus (3.3-6.3 356 mean ingested fish mOTUs per individual) but generally low for all other taxa (0.9-2.4 mean 357 ingested fish mOTUs per individual). 
360 Table 1: Diet richness in mOTUs, expressed for the whole diet and the fish fraction, both at

361 the population (total) and individual level (Mean \pm SE), and network centrality metrics

362 (weighted in-degree and eigenvector centrality) for each consumer. *Centrality values of both

363 echinoids are those of the mOTU 'Echinoidea' since they could not be differentiated during

364 metabarcoding analysis.

\begin{tabular}{|c|c|c|c|c|c|c|c|c|c|c|}
\hline \multirow[b]{2}{*}{ Class } & \multirow[b]{2}{*}{ Species } & \multirow[b]{2}{*}{$\mathrm{N}$} & \multicolumn{3}{|c|}{ Total richness } & \multicolumn{3}{|c|}{ Fish richness } & \multicolumn{2}{|c|}{ Centrality metrics } \\
\hline & & & Total & Mean & $\mathrm{SE}$ & Total & Mean & SE & $\begin{array}{l}\text { Weighted } \\
\text { in-degree }\end{array}$ & $\begin{array}{l}\text { Eigen } \\
\text { vector }\end{array}$ \\
\hline \multirow{9}{*}{ Actinopterygii } & Callionymus lyra & 10 & 26 & 5.1 & 0.7 & 4 & 0.9 & 0.3 & 31.3 & 0.49 \\
\hline & Chelidonichthys lucerna & 23 & 24 & 2.9 & 0.5 & 12 & 1.0 & 0.3 & 43.4 & 0.45 \\
\hline & Conger conger & 19 & 25 & 4.1 & 0.3 & 14 & 1.6 & 0.3 & 29 & 0.63 \\
\hline & Merlangius merlangus & 20 & 23 & 3.5 & 0.5 & 11 & 2.3 & 0.3 & 26.1 & 0.60 \\
\hline & Merluccius merluccius & 19 & 16 & 2.5 & 0.3 & 9 & 1.7 & 0.2 & 120 & 0.78 \\
\hline & Pleuronectes platessa & 20 & 24 & 5.5 & 0.5 & 8 & 1.7 & 0.3 & 25.8 & 0.38 \\
\hline & Pollachius pollachius & 17 & 36 & 5.4 & 0.7 & 13 & 2.4 & 0.5 & 20.6 & 0.34 \\
\hline & Trachurus trachurus & 25 & 34 & 7.4 & 0.5 & 15 & 4.6 & 0.3 & 93.8 & 0.71 \\
\hline & Trisopterus luscus & 20 & 33 & 4.2 & 0.4 & 11 & 1.4 & 0.2 & 84.2 & 0.84 \\
\hline Cephalopoda & Sepia officinalis & 27 & 38 & 4.6 & 0.4 & 24 & 3.3 & 0.5 & 1.7 & 0.10 \\
\hline \multirow{2}{*}{ Chondrichthyes } & Raja undulata & 21 & 21 & 4.3 & 0.4 & 13 & 2.2 & 0.2 & 19.6 & 0.56 \\
\hline & Scyliorhinus canicula & 19 & 39 & 7.0 & 0.8 & 16 & 2.4 & 0.5 & 11.6 & 0.46 \\
\hline \multirow{2}{*}{ Echinoidea } & Echinus esculentus & 1 & 16 & 16.0 & NA & 9 & 9.0 & NA & $0(7.9 *)$ & $0(0.23 *)$ \\
\hline & Psammechinus miliaris & 19 & 57 & 6.4 & 0.9 & 18 & 2.3 & 0.5 & $0(7.9 *)$ & $0(0.23 *)$ \\
\hline Gastropoda & Buccinum undatum & 1 & 4 & 4.0 & NA & 1 & 1.0 & NA & 4.7 & 0.13 \\
\hline \multirow{6}{*}{ Malacostraca } & Atelecyclus undecimdentatus & 20 & 26 & 4.1 & 0.6 & 18 & 2.2 & 0.6 & 170.5 & 0.59 \\
\hline & Cancer pagurus & 17 & 25 & 3.5 & 0.5 & 16 & 1.9 & 0.5 & 0 & 0 \\
\hline & Crangon sp. & 13 & 25 & 7.9 & 1.4 & 19 & 6.3 & 1.3 & 147 & 0.50 \\
\hline & Maja brachydactyla & 23 & 18 & 2.1 & 0.4 & 8 & 0.6 & 0.2 & 0 & 0 \\
\hline & Necora puber & 16 & 25 & 4.2 & 0.7 & 16 & 2.2 & 0.5 & 94.6 & 0.82 \\
\hline & Paguroidea & 15 & 22 & 3.8 & 0.5 & 11 & 1.1 & 0.3 & 8.9 & 0.27 \\
\hline Polychaeta & Aphrodita aculeata & 4 & 6 & 2.3 & 0.6 & 2 & 1.0 & 0.4 & 0 & 0 \\
\hline
\end{tabular}

366 
Weighted percentage of occurrence (wPOO) of fish mOTUs in the diet of the different consumers varied from a minimum of $15.9 \%$ in $M$. brachydactyla to a maximum of $77 \%$ in Merluccius merluccius (Table S5). Fish represented $\geq 50 \%$ of all ingested mOTUs in terms of wPOO in the fishes Merlangius merlangus, M. merluccius, T. trachurus and $R$. undulata, in the 371 cuttlefish S. officinalis, but also in the echinoid E. esculentus, the shrimp Crangon sp. and the 372 polychaete $A$. aculeata (Fig. 1; Fig. S1).

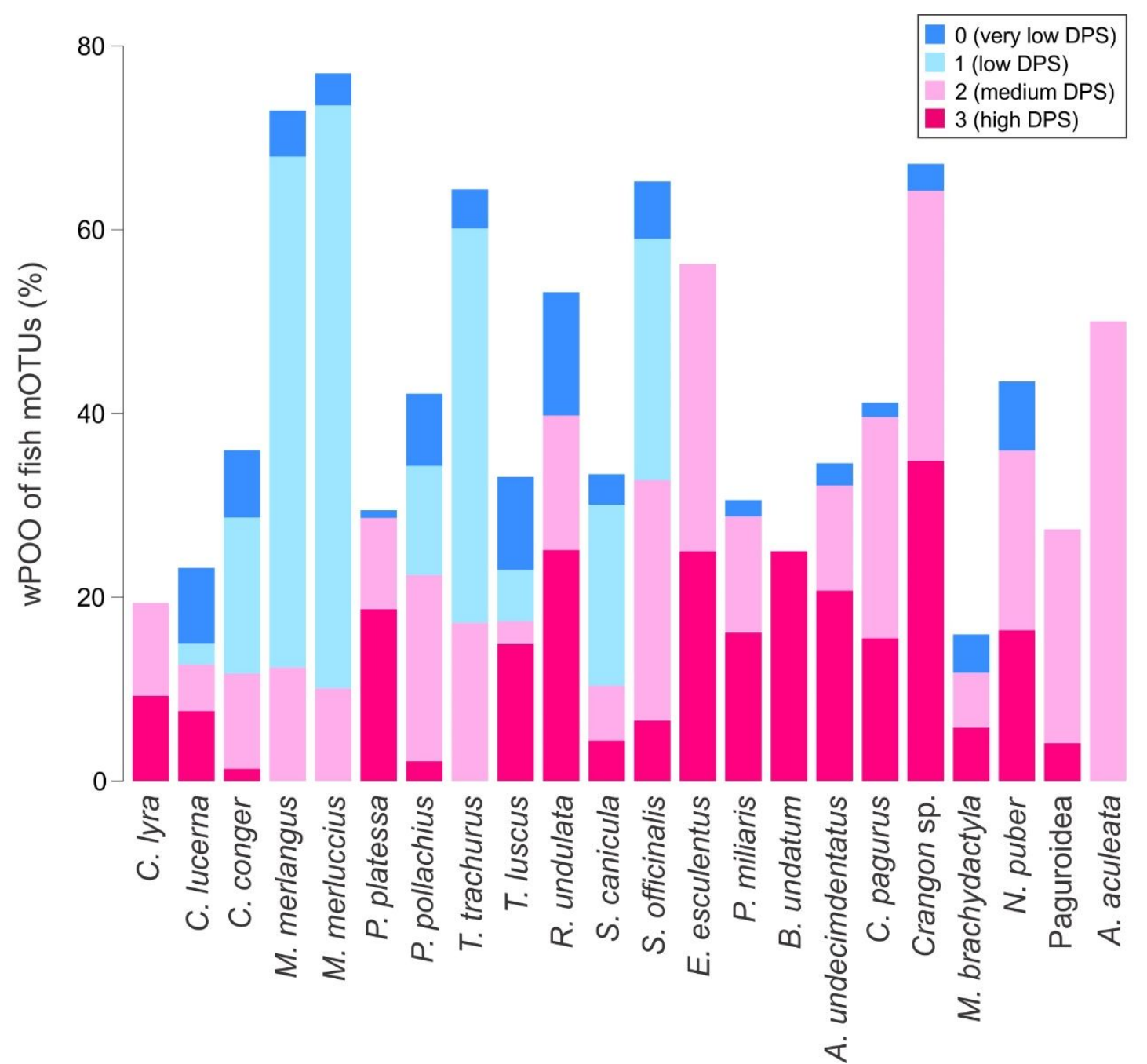

Figure 1: Weighted percentage of occurrence (wPOO [\%]) of the fish mOTUs (Actinopterygii 376 and Chondrichthyes) identified via gut contents metabarcoding of the 22 consumers. Fish 377 mOTUs are categorized according to the 'discard probability score' (DPS), from 0 (dark blue) $378=$ very low probability to 3 (dark pink) $=$ high probability of being ingested as fisheries discard. 


\section{Potential importance of discarded fish ingestion across consumer taxa}

381 Prey with the highest discard probability score (DPS $=3$ ) were found in the gut contents of all 382 consumers, except the three predatory fishes M. merlangus, M. merluccius and T. trachurus, 383 and the polychaete $A$. aculeata. Fish prey that have a medium to high probability of having 384 been consumed as fisheries discards (i.e. DPS $\geq 2$ ) were found in the gut contents of all 22 385 consumers. We considered that the fish fraction of the diet of a species was dominated by 386 potential discard ingestions when mOTUs with DPS $\geq 2$ represented more than $50 \%$ of all 387 ingested fish mOTUs in terms of wPOO. All consumer taxa had a fish fraction of the diet 388 dominated by prey with DPS $\geq 2$, except the five predatory fishes Conger conger, $M$. 389 merlangus, M. merluccius, T. trachurus and S. canicula. In these five species, fish fraction of 390 the diet was dominated by species belonging to the family Clupeidae, which were being 391 discarded by local fisheries at the time of sampling according to our observations but are also 392 known to be naturally predated upon by those consumers, hence their classification as DPS $=1$ (Table S4 and S4). However, in T. trachurus, fish prey with DPS = 2 still constituted 'typical' 394 prey (i.e. dominant items in terms of frequency of occurrence: $\mathrm{FOO}=80 \%$ ). Among fishes, the 395 species that included the most prey with DPS $\geq 2$ relative to other fish prey were C. lyra, 396 Pleuronectes platessa and R. undulata (100\%, 97.2\% and 74.8\% of their fish diet respectively). 397 All invertebrates excluding S. officinalis had a fish fraction of the diet almost entirely composed 398 of prey with DPS $\geq 2(73.9-100 \%$ of fish mOTUs) which were also generally largely dominant 399 in terms of FOO, indicative of a reliance on fish prey potentially mainly linked to scavenging 400 on discards (Fig. 1; Table S5). Prey with DPS $\geq 2$ represented 11.8 to $56.3 \%$ of the whole diet 401 of these species. Fish fraction of the diet was entirely constituted of prey with DPS $\geq 2$ in $E$. 402 esculentus, Paguroidea and A. aculeata constituting 56.3\%, 27\% and 50\% of their whole diet, 403 respectively. A. aculeata, Paguroidae, M. brachydactyla and S. officinalis had the lowest 
404 reliance on prey with DPS $=3$ relative to their whole diet $(0-6.6 \%$ wPOO). Conversely, 405 echinoids and all other decapods relied importantly on prey with DPS $=3$, reaching 15.5 to $40634.9 \%$ of their whole diet. The gastropod B. undatum relied exclusively on fish with DPS $=3$, 407 constituting $25 \%$ of its whole diet.

408 409

\section{Molecular ecological network analysis}

410 Diet information were used to model a network of trophic interactions among the 22 sampled 411 consumers and their prey, totaling 563 weighted links (trophic interactions) between 159 nodes 412 (sampled consumers and mOTUs) (Fig. 2; Figure S2). Among the 563 trophic links, 48\% (268 413 links) involved fish prey, that is $12 \%$ with DPS $=3,19 \%$ with DPS $=2,10 \%$ with DPS $=1$ and $4147 \%$ with DPS $=0$. Among these 270 trophic links involving fish prey, $66 \%$ concerned fish prey 415 that had a medium to high probability of having been consumed as fisheries discards (DPS $\geq$ 416 2); $40 \%$ with DPS $=2$ and $26 \%$ with DPS $=3$ relative to the consumer. Both centrality metrics 417 provided complementary information identifying species whose fish fraction of the diet could 418 be dominated by discards, or fish that were themselves importantly discarded, as functionally 419 important nodes in the modeled interaction network (Table 1; Table S6). Among taxa with the 420 highest weighted in-degree centrality values, we found species whose fish fraction of the diet 421 could be potentially largely dominated by discards (e.g. the decapods Atelecyclus 422 undecimdentatus, Crangon sp. and Necora puber). The fish with the highest weighted in-degree centrality values were all part of species discarded by the commercial fleet in the area and at

424 the time of sampling: T. trachurus, Trisopterus luscus and C. lucerna which were observed 425 among the most discarded species, as well as M. merluccius and multiple Clupeidae which were 426 observed to be discarded in lower quantities. A similar pattern was visible in Eigenvector 427 centrality values, but with an increased importance of fish, mainly Clupeidae and T. luscus, and 428 a slight decrease of decapods importance in the network (Table 1; Table S6). 


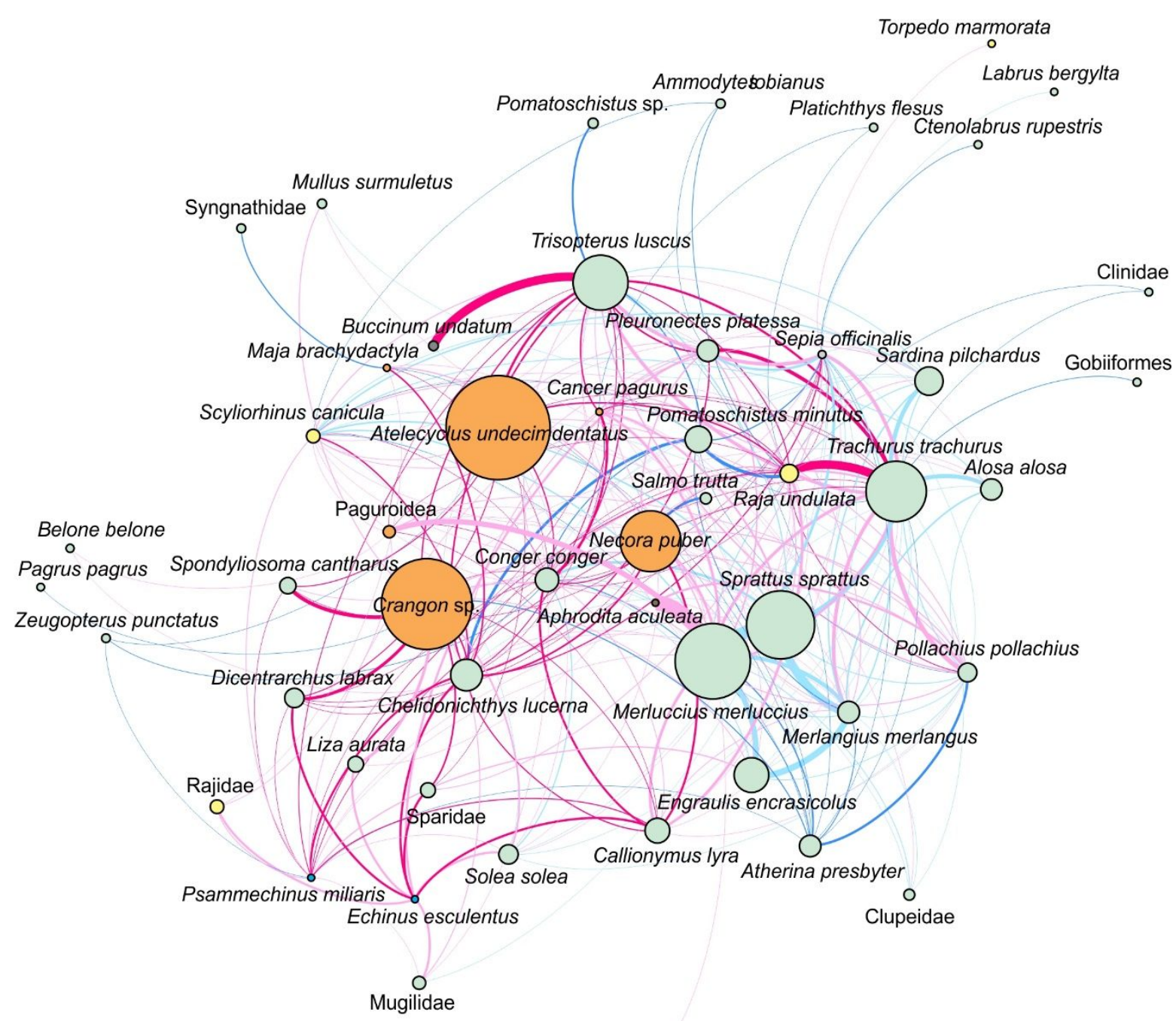

Node

\begin{tabular}{|l|c|}
\hline Taxonomic affiliation & Size (weighted in-degree) \\
Actinopterygii & \\
Chondrichthyes & \\
Cephalopoda & \\
Echinoidea \\
Gastropoda \\
Malacostraca \\
Polychaeta
\end{tabular}

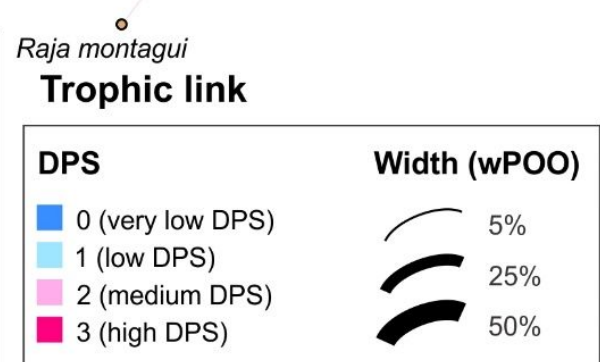

431 Figure 2: Subset of the molecular network constructed from gut content metabarcoding data, 432 depicting trophic interactions among the 22 consumers and their prey; only the trophic links 433 involving fish prey are presented, see Fig. S2 for the complete network. Nodes are representing 434 the consumers and prey mOTUs. Node color depicts taxonomic affiliation (by Class). Node 435 size is proportional to its importance as a food source for other taxa (weighted in-degree 436 centrality). Link width is proportional to wPOO of prey and color varies according to prey DPS. 


\section{Discussion}

\section{Importance of discard consumption across marine fauna}

440 With the introduction of the LO in European waters (European Union 2013), the amount of

441 fisheries discards is expected to decline. However, it is not clear to which extent scavenging 442 behaviors may be common and determine the diet of marine fauna (e.g. Bluhm \& Bechtel, 2003;

443 Olaso et al., 2002; Ramsay et al., 1997), especially in fishing grounds subject to important 444 discarding activity. Here, we showed that potential ingestion of discarded fish was generalized 445 from low trophic level benthic invertebrates to predatory fishes in shallow coastal fishing 446 grounds of the Bay of Bourgneuf, with a fish fraction of the diet likely dominated by potential 447 discards in 17 of the 22 sampled taxa. Diet involving prey with DPS $\geq 2$ generally corresponded 448 to prey that may not be naturally predated upon. For example, gut content metabarcoding 449 revealed that $30 \%$ of Callionymus lyra, a small coastal benthivore fish that feeds mainly on 450 benthic invertebrates (Fricke 1986; Thompson et al. 2020), ingested Conger conger and 451 Merluccius merluccius, which are larger and higher in the food chain (Arroyo et al. 2019). 452 These ingestions accounted for $100 \%$ of the fish diet of this species. As both C. conger and $M$. 453 merluccius are eviscerated on board, the ingestion of these two species by $C$. lyra likely 454 corresponds to eating discarded viscera. Note that some fish mOTUs identified in the guts of 455 some species might correspond to predation on eggs or early life stages which could be 456 underassessed in the diet literature, possibly introducing a bias in the interpretation of high DPS 457 prey consumption. However, the trophic guild approach included in the calculation of the DPS 458 (Thompson et al. 2020) is based on large databases of stomach content records which also 459 report eggs and larvae consumption (i.e. the 'Integrated Database and Portal for Fish Stomach 460 Records' [DAPSTOM; Pinnegar 2014]) and ICES 'Year of the Stomach' database [ICES 461 1997]), which should limit such bias as much as possible. 
Large-scale studies have provided contrasting results regarding the importance of 463 discards for scavengers concluding to either influential (e.g. Catchpole \& Frid 2006) or limited 464 effects (e.g. Depestele et al. 2019), but calling for more empirical studies in diverse 465 environmental settings. In invertebrates, fish fraction of the diet was largely dominated by prey 466 with DPS $\geq 2$ (74-100\%), suggesting that all invertebrates sampled may rely mainly on discards 467 concerning fish prey. This is consistent with the fact that most of these species, except Sepia 468 officinalis, are unlikely to catch and feed upon live fish. Although some are known 469 necrophageous, the proportion of fish ingestion likely originating from discarding was 470 particularly high in the crabs Cancer pagurus, Necora puber and Atelecyclus undecimdentatus. 471 Previous studies recorded discard consumption by Buccinum undatum (Evans et al., 1996), sea 472 urchins (González-Irusta et al. 2014), crabs and spider crabs (Ramsay et al. 1997; Bozzano \& 473 Sardà 2002). While we judged that Aphrodita aculeata, Echinus esculentus and Buccinum 474 undatum have too low sample sizes to allow for any generalization of findings, their reporting 475 remain valuable regarding the paucity of information on the diet of these species. Overall, these 476 results suggest that in coastal fishing grounds subject to important discarding activity, the 477 reliance of local invertebrate macrofauna on fish likely ingested as discards may be important, 478 and reveal scavenging behaviors that appear to be under documented or whose strengths may 479 be underappreciated.

480 In fish, potential discarded fish ingestions were more variable across taxa and less 481 important in more predatory fishes compared to others. However, this may also be due to the 482 fact that in predatory fishes for which piscivory is well established in the literature, such as 483 Conger conger, Merlangius merlangus, Merluccius merluccius, Trachurus trachurus and 484 Scyliorhinus canicula (See Table S4 for references), most of the ingested fish mOTUs were 485 known to be naturally predated upon, therefore lowering their DPS according to our 486 methodology, even if they were importantly discarded in the area. A previous study by Olaso 
487 et al. (2002) acknowledged important consumption of discarded fish such as Clupeidae by $S$. 488 canicula, while another study by Groenewold and Fonds (2000) evidenced that M. merlangus 489 were attracted by fish carcasses. Fishes that should occupy lower trophic levels (Callionymus 490 lyra, Chelidonichthys lucerna, Pleuronectes platessa, Pollachius pollachius, Trisopterus luscus 491 and Raja undulata; Arroyo et al., 2019) showed unexpectedly important consumption of fish 492 potentially linked to scavenging on discards (DPS $\geq 2$ ). Such scavenging behaviors were 493 already noted for Rajidae (Olaso et al. 2002), as well as for P. platessa which were documented 494 waiting and feeding at the chelae of crabs around discarded fish (Jenkins et al. 2004). C. lyra, 495 C. lucerna, P. platessa and T. luscus also showed attraction towards fish carcasses in the study 496 by Groenewold and Fonds (2000). Our study suggests that $R$. undulata, P. platessa, T. luscus 497 and C. lyra may be the main consumers of discards in the fish community.

498

\section{Potential consequences of the Landing Obligation suggested by network}

\section{analysis}

501 Studying food web structure helps forecasting the impact of trophic changes on ecosystem 502 functioning (Allesina \& Pascual 2009; Meyer et al. 2020). Because of the lack of infrastructure 503 on the ground, some areas benefitted from an extension of the application of the Landing 504 Obligation (LO) to 2020, as is the case of the Bay of Biscay (European Union 2019), explaining 505 that species under total allowable catches (TAC) and quota were still being discarded at the 506 time of study. This provided the opportunity to picture the structure of a network of trophic 507 interactions among species feeding in coastal fishing grounds subject to important discarding 508 and their prey. Network analysis revealed that in the studied fishing ground, $66 \%$ of the trophic 509 links implying fish prey potentially involved discard consumption (DPS $\geq 2$ ), including 26\% 510 with the highest discard probability score $(\mathrm{DPS}=3)$. This provides novel insights suggesting 
511 that discarded fish consumption might constitute a non-negligible factor affecting the structure 512 of the local food web and community.

One may argue that discards might enhance secondary production in marine food webs

514 (Bluhm \& Bechtel 2003), in which case a reduction of discarding could lead to negative effects 515 on populations most supported by them and to associated indirect effects. It is worth noting that 516 not all discarded species are subject to the LO, implying that not all discarding will end with its 517 full application in the area, but only those species under TAC and quota. Likewise, even if 518 discarding is regulated, consumption of discarded viscera should still be possible since large 519 catches such as C. conger or M. merluccius would continue to be eviscerated on board. 520 However, species subject to the LO represented an important fraction of ingested fish in the 521 sampled community (e.g. Trachurus trachurus, Engraulis encrasicolus, Merluccius merluccius 522 or the different rays; see all species under TAC and quota among our samples in Table S5). 523 Results show that decapods such as Atelecyclus undecimdentatus, Crangon sp. and Necora 524 puber, or the fish Trisopterus luscus which relied potentially importantly on discards, but also 525 Trachurus trachurus which was amongst the most discarded fish according to both our 526 observations and OBSMER data, constitute some of the most important structuring nodes in 527 the network. This implies that they may potentially strongly affect the local abundance of other 528 taxa via bottom-up effects (Elhesha et al. 2017). This is consistent with studies describing the 529 central role of decapods, including as food sources for diverse taxa (Boudreau \& Worm 2012) 530 and the fact that decapods seem to constitute the dominant invertebrate Order attracted by or 531 consuming discards (Depestele et al. 2019). By reducing the amount of food supporting these 532 central species or reducing their discarding, the LO may potentially trigger indirect effects, 533 altering the local network of trophic interactions and community structure. Another 534 consequence that is perhaps more complex to predict is the potential positive effect on fish 535 populations which were previously strongly subject to discarding. On the one hand they could 
536 provide more food for species higher in the food chain, following natural predation. On the 537 other hand they could increase predation pressure on species lower in the food chain and modify 538 food web structure via top-down effects. For example, a discard ban on Trachurus trachurus may positively affect its population, increasing predation pressure on the variety of Clupeidae

The 'snapshot' character of trophic information obtained via gut content metabarcoding and the focus on a limited number of species corresponding to those targeted by the local commercial trawling fleet may not allow to fully apprehend the complex consequences of the trawling) may introduce a bias in the representation of species against pelagic ones while 548 focusing on the bentho-demersal assemblage. However, this also reflects the specific environmental context in which the study took place, which is a shallow coastal fishing ground (4-30m depth) representative of many coastal fisheries in the South of Europe. The presence of T. trachurus in our dataset shows that some pelagic species can nonetheless feed in such fishing 552 grounds, underlying the increased bentho-pelagic coupling in shallow coastal areas (Kopp et al. 2015). More precise inference on the effects of discard reduction on the pelagic compartment 554 of the food web could be drawn from conducting complementary studies using a larger variety 555 of capture methods (e.g. including gill-nets, traps), as well as replicate our study design in 556 deeper and open-water fisheries. Studies conducted in deep-sea offshore fisheries (200-800 m 557 depth) dominated by trawling suggest a similar pattern whereas important quantities of discards 558 end up on the seabed and be consumed by various benthic fauna, including small crustaceans 559 which are important prey of targeted commercial species (Castro, Araújo \& Monteiro 2015). 560 Offshore open-water fisheries may be dominated by other métiers (e.g. gill-nets, longlines) 
561 which often target single species, generate less discards (Cornou et al. 2021) and operate in a 562 spottier fashion than in coastal fisheries which are more geographically comprehensively 563 fished. In these contexts, the possibility of consumption by large pelagic predators before 564 discards reach the bottom might reduce its integration into the benthic food web compared to 565 our study system and encounter probability (i.e. the likelihood to come across fisheries discards) 566 might be reduced as well for both pelagic and bentho-demersal species (Depestele et al. 2019), 567 possibly leading to a more diluted impact across the whole food web. Yet, we believe that more 568 empirical studies are needed to clarify and quantify trophic links between marine fauna and 569 discards in these structurally more complex systems, which would require a wider array of 570 capture methods to be deployed to cover both pelagic and benthic species.

\section{DNA metabarcoding to advance research on discards reintegration into marine}

573 food webs

Gut content metabarcoding promises to revolutionize food web studies by yielding 575 unparalleled resolution of trophic interactions (Casey et al. 2019; Takahashi et al. 2020), but 576 limitations should also be considered in order to advance research on discards reintegration into 577 marine food webs. It was successfully used to identify species potentially originating from 578 fisheries discards in the diet of Albatrosses (Mcinnes et al. 2017) and quantify discard 579 consumption in marine fauna in the present study. Here, gut content metabarcoding allowed to 580 identify 154 mOTUs (considering a $\geq 1 \%$ relative read abundance threshold; Deagle et al. 581 2019), with 40 belonging to Actinopterygii and Chondrichthyes, which represents an 582 unprecedented resolution in a demersal food web. Yet, taxonomic levels were sometimes 583 dropped from species to genus, or further, whenever the similarity between primary and 584 secondary reference sequences was equal or higher to the similarity between the primary 585 reference and the query sequence during taxonomic assignment, resulting in sequence 
586

587

588

589

590

591

592

593

594

595

596

597

598

599

600

601

602

603

604

605

606

607

608

609

610

assignment to the most recent common ancestor. Such cases can occur because of incomplete reference databases. Improving the local reference database is therefore crucial to maximize the resolution of taxonomic identification (Casey et al. 2019). It is also worth noting that while the $18 \mathrm{~S}$ rDNA region may provide an exceptional coverage across the prey items of interest in this study, it may provide poorly reliable taxonomic resolution. The use of complementary markers can improve taxonomic resolution within specific groups of interest (da Silva et al. 2019). Here, the complementary use of Tele 02 (targeting $12 \mathrm{~S}$ region) helped improve the resolution of fish mOTUs identification compared to Euka02 alone (targeting 18S region): Among the 40 fish mOTUs identified, 32 were assigned to species level, 1 to genus, 6 to family and 1 to order. Since taxonomic richness is dependent upon clustering level, taxonomic richness of the whole diet may be influenced by lower or variable resolution yielded by Euka02 for non-fish groups. Taxonomic richness of the fish fraction of the diet should be much less influenced by such problem. Specific markers for other taxonomic groups should similarly improve identification resolution. Using multiple markers could also help limit the intrinsic biases associated with primer choice and difference in amplification rate among sequences (Deagle et al., 2014).

One intrinsic limitation of applying metabarcoding to study diet is that one cannot decipher between conspecifics consumption and DNA coming from the consumer's own degrading cells, which is the reason for removing 'self-hits' from diet data (i.e. DNA of the consumer within its own gut content) (Taberlet et al. 2018; Casey et al. 2019). We found no evidence of an important role of cannibalism in the trophic ecology of the studied species based on gut content data from large databases used for DPS calculation. But beyond cannibalism of living conspecifics, one way self-hits censoring might still impact the estimated diet is by making a part of discard consumption invisible and therefore unaccounted for, namely if a species is consuming discarded conspecifics. This might be more impactful for species that are importantly discarded in the area (e.g. C. conger, T. trachurus, T. luscus), as one would expect 
611 the probability of consuming discarded conspecifics to increase with discarding rate of that 612 species. Important amount of consumer's DNA in a sample might impede the ability of PCR to 613 reveal the true diversity of the diet because of a quick saturation of the reaction. But in our 614 dataset self-hit rate was generally low, with only five fish species out of twenty-two depicting 615 high self-hit rates with Tele02. High read counts of only their own DNA can be explained by 616 the absence of fish consumption, which is confirmed by the absence of (other) fish mOTUs 617 identified with Euka02. In this specific case, self-hits have little impact on diet estimates.

618 Finally, like traditional morphological identification, gut content metabarcoding only 619 provides dietary information on a short time-scale. However, its sensitivity to identify highly 620 degraded prey from intestinal content allows to access diet information that is often missed in 621 traditional morphological identification (Takahashi et al. 2020). The use of long-term 622 biomarkers such as stable isotopes or fatty acids as complementary tools may allow to decipher 623 between opportunistic scavenging and specialized long-term strategies across the community, 624 and better apprehend the potential consequences of the LO.

\section{Conclusions}

627 Ultimately, reducing bycatch and discarding remain conservation priorities, but it appears also 628 crucial to understand and anticipate the potential consequences of reducing discards for the 629 variety of marine species that may have become quite dependent upon them. Our results reveal 630 that consumption of fish likely ingested as discards was important and generalized across 631 marine fauna feeding in fishing grounds subject to important discarding, and identified that 632 discards may support multiple species potentially playing an important structuring role in the 633 local food web, such as decapods. DNA metabarcoding of gut contents, together with reliant 634 methodologies to establish the probability of prey to originate from discarding, offers the 635 possibility to quickly assess dependence of a community on fisheries discards. These results 
636 could be further confirmed using stable isotopes or fatty acid tracers and repeated in various environmental settings to be implemented in ecosystem models such as Ecopath with ECOSIM in which trophic links with discards and corresponding energy fluxes may currently be underestimated.

\section{Authors' contributions}

DK, MM and SM designed the study and methodology; DK and SM sampled and preserved gut contents; SM realized the observations on discard frequencies; BL participated to sequence curation and analysed metabarcoding data; BL led the writing of the manuscript. All authors contributed critically to the drafts and gave final approval for publication.

\section{Acknowledgements}

648 This study was part of the DREAM project supported by the European Maritime and Fisheries 649 Fund (EMFF) and France Filière Pêche. The authors thank the crew of the fishing vessel, as 650 well as the three scientists Fabien Morandeau, Nicolas Goascoz and Julien Simon for their help 651 to conduct the onboard experiments. The authors are deeply indebted to the French Directorate 652 of Marine Fisheries and Aquaculture (DPMA), to the team from the IFREMER French 653 Fisheries Information System (SIH) for providing access to the OBSMER database and to Loïc 654 Baulier who processed the data.

\section{References}

657 Allesina, S. \& Pascual, M. (2009) Googling food webs : can an eigenvector measure species' 658 importance for coextinctions ? PloS Computational Biology, 5, e1000494.

659 Arroyo, N.-L., Safi, G., Vouriot, P., López-López, L., Niquil, N., Le Loc’h, F., Hattab, T. \& Preciado, I. (2019) Towards coherent GES assessments at sub-regional level: signs of 
661

662

663

664

665

666

667

668

669

670

671

672

673

674

675

676

677

678

679

680

681

682

683

684

685

fisheries expansion processes in the Bay of Biscay using an OSPAR food web indicator, the mean trophic level. ICES Journal of Marine Science, 76, 1543-1553.

Bascompte, J., Melián, C.J. \& Sala, E. (2005) Interaction strength combinations and the overfishing of a marine food web. Proceedings of the National Academy of Sciences, 102, 5443-5447.

Bastian, M., Heymann, S. \& Jacomy, M. (2009) Gephi: an open source software for exploring and manipulating networks. International AAAI Conference on Weblogs and Social Media

Bluhm, B.A. \& Bechtel, P.J. (2003) The potential fate and effects of seafood processing wastes dumped at sea: a review. Advances in Seafood Byproducts, Alaska Sea Grant College Program (ed P.J. Bechtel), pp. 121-140. University of Alaska, Fairbanks, AK.

Boudreau, S.A. \& Worm, B. (2012) Ecological role of large benthic decapods in marine ecosystems : a review. Marine Ecology Progress Series, 469, 195-213.

Boyer, F., Mercier, C., Bonin, A., Le Bras, Y., Taberlet, P. \& Coissac, E. (2015) OBITools: a unix-inspired software package for DNA metabarcoding. Molecular Ecology Resources, 16, 176-182.

Bozzano, A. \& Sardà, F. (2002) Fishery discard consumption rate and scavenging activity in the northwestern Mediterranean Sea. ICES Journal of Marine Science, 59, 15-28.

Calderón-Sanou, I., Münkemüller, T., Boyer, F., Zinger, L. \& Thuiller, W. (2019) From environmental DNA sequences to ecological conclusions : How strong is the influence of methodological choices ? Journal of Biogeography, 47, 193-206.

Casey, J.M., Meyer, C.P., Morat, F., Brandl, S.J., Planes, S. \& Parravicini, V. (2019) Reconstructing hyperdiverse food webs : Gut content metabarcoding as a tool to disentangle trophic interactions on coral reefs. Methods in Ecology and Evolution, 10, $1157-1170$. 
686 Castro, M., Araújo, A. \& Monteiro, P. (2015) Fate of discards from deep water crustacean 687 trawl fishery off the south coast of Portugal. New Zealand Journal of Marine and Freshwater Research, 39, 437-446.

Catchpole, T. \& Frid, C.L.J. (2006) Importance of discards from the English Nephrops norvegicus fishery in the North Sea to marine scavengers. Marine Ecology Progress Series, 313, 215-226.

692 Coissac, E. (2012) Oligotag: a program for designing sets of tags for next-generation 693 sequencing of multiplexed samples. Data Production and Analysis in Population

Genomics, Methods in Molecular Biology Series (eds F. Pompanon \& A. Bonin), pp. 1331. Humana Press, Totowa, New Jersey.

696 Cornou, A., Quinio-scavinner, M., Sagan, J., Cloâtre, T., Dubroca, L. \& Billet, N. (2021) 697 Captures et Rejets Des Métiers de Pêche Français. Résultats Des Observations à Bord Des Navires de Pêche Professionnelle En 2019. Obsmer.

699 Deagle, B.E., Clarke, L.J., Thomas, A.C., Mcinnes, J.C., Vesterinen, E.J., Clare, E.L., 406.

Deagle, B.E., Jarman, S.N., Coissac, E., Pompanon, F. \& Taberlet, P. (2014) DNA metabarcoding and the cytochrome c oxidase subunit I marker : not a perfect match. Biology letters, 10, 20140562.

Depestele, J., Feekings, J., Reid, D.G., Cook, R., Gascuel, D., Girardin, R., Heath, M., Hernvann, P.-Y., Morato, T., Soszynski, A. \& Savina-Rolland, M. (2019) The Impact of Fisheries Discards on Scavengers in the Sea. The European Landing Obligation: Reducing Discards in Complex, Multi-Species and Multi-Jurisdictional Fisheries (eds 
Publishing, Cham.

Elhesha, R., Kahveci, T. \& Baiser, B. (2017) Motif centrality in food web networks. Journal of Complex Networks, 5, 641-664.

European Union. (2013) Regulation (EU) No 1380/2013 of the European parliament and of the council of 11 December 2013 on the Common Fisheries Policy amending Council Regulations (EC) No 1954/2003 and (EC) No 1224/2009 and repealing Council Regulations (EC) No 2371/2002 and (EC). Official Journal of the European Union, L354, 22-61.

European Union. (2019) Commission delegated regulation (EU) 2019/2237 of 1 October 2019 specifying details of the landing obligation for certain demersal fisheries in SouthWestern waters for the period 2020-2021. Official Journal of the European Union, L336, $26-33$.

Evans, P.L., Kaiser, M.J. \& Hughes, R.N. (1996) Behaviour and energetics of whelks, Buccinum undutum (L .), feeding on animals killed by beam trawling. Journal of Experimental Marine Biology and Ecology, 197, 51-62.

Ficetola, G.F., Coissac, E., Zundel, S., Riaz, T. \& Shehzad, W. (2010) An In silico approach for the evaluation of DNA barcodes. BMC Genomics, 11, 434.

Fricke, R. (1986) Callionymidae. Fishes of the North-eastern Atlantic and the Mediterranean (eds P.J.P. Whitehead, M.-L. Bauchot, J.-C. Hureau, J. Nielsen \& E. Tortonese), pp. 1086-1093. UNESCO, Paris.

Garthe, S., Camphuysen, K.C. \& Furness, R.W. (1996) Amounts of discards by commercial fisheries and their significance as food for seabirds in the North Sea. Marine Ecology Progress Series, 136, 1-11.

Gauduchon, T., Cornou, A., Quinio-Scavinner, M., Goascoz, N., Dubroca, L. \& Billet, N. (2020) Captures et Rejets Des Métiers de Pêche Français. Résultats Des Observations à 
Bord Des Navires de Pêche Professionnelle En 2018. Obsmer.

737

738

739

740

González-Irusta, J.M., Preciado, I., López-López, L., Punzón, A., Cartes, J.E. \& Serrano, A. (2014) Trawling disturbance on the isotopic signature of a structure-building species, the sea urchin Gracilechinus acutus (Lamarck, 1816). Deep-Sea Research Part II: Topical Studies in Oceanography, 106, 216-224.

Groenewold, S. \& Fonds, M. (2000) Effects on benthic scavengers of discards and damaged benthos produced by the beam-trawl fishery in the southern North Sea. ICES Journal of Marine Science, 57, 1395-1406.

Guardiola, M., Uriz, M.J., Taberlet, P., Coissac, E., Wangensteen, S. \& Turon, X. (2015) Deep-sea, deep-sequencing: metabarcoding extracellular DNA from sediments of marine canyons. PLoS ONE, 10, e0139633.

Guillen, J., Holmes, S.J., Carvalho, N., Casey, J., Dörner, H., Gibin, M., Mannini, A., Vasilakopoulos, P. \& Zanzi, A. (2018) A review of the European union landing obligation focusing on its implications for fisheries and the environment. Sustainability, 10, 900 .

Hill, B.J. \& Wassenberg, T.J. (2000) The probable fate of discards from prawn trawlers fishing near coral reefs A study in the northern Great Barrier Reef, Australia. Fisheries Research, 48, 277-286.

ICES. (1997) Database Report of the Stomach Sampling Project 1991.

Ifremer. Système d'Informations Halieutiques. (2020) Quartier Maritime Noirmoutier. 2019. Activité Des Navires de Pêche.

Jackson, J.B.C., Kirby, M.X., Berger, W.H., Bjorndal, K.A., Botsford, L.W., Bourque, B.J., Bradbury, R.H., Cooke, R., Erlandson, J., Estes, J.A., Hughes, T.P., Kidwell, S., Lange, C.B., Lenihan, H.S., Pandolfi, J.M., Peterson, C.H., Steneck, R.S., Tegner, M.J. \& Warner, R.R. (2001) Historical overfishing and the recent collapse of coastal 
ecosystems. Science, 293, 629-638.

Jacomy, M., Venturini, T., Heymann, S. \& Bastian, M. (2014) ForceAtlas2 , a continuous graph layout algorithm for handy network visualization designed for the Gephi software. PLoS ONE, 9, e98679.

Jenkins, S.R., Mullen, C. \& Brand, A.R. (2004) Predator and scavenger aggregation to discarded by-catch from dredge fisheries : importance of damage level. Journal of Sea Research, 51, 69-76.

Karris, G., Rinis, V.K., Kalogeropoulou, A., Xirouchakis, S., Machias, A., Maina, I. \& Kavadas, S. (2018) The use of demersal trawling discards as a food source for two scavenging seabird species : a case study of an eastern Mediterranean oligotrophic marine ecosystem. Avian Research, 9, 26.

Kelleher, K. (2005) Discards in the World's Marine Fisheries. An Update. FAO Fisheries Technical Paper. No. 470, Rome.

Kopp, D., Lefebvre, S., Cachera, M., Villanueva, M.C. \& Ernande, B. (2015) Reorganization of a marine trophic network along an inshore-offshore gradient due to stronger pelagicbenthic coupling in coastal areas. Progress in Oceanography, 130, 157-171.

Kopp, D., Robert, M., Chouvelon, T. \& Méhault, S. (2016) Some expected impacts of the Common Fishery Policy on marine food webs. Marine Policy, 66, 8-14.

MarLIN. (2006) BIOTIC - Biological Traits Information Catalogue. URL www.marlin.ac.uk/biotic [accessed 4 February 2021]

Mcinnes, J.C., Jarman, S.N., Lea, M., Raymond, B., Deagle, B.E., Phillips, R.A., Catry, P., Stanworth, A., Weimerskirch, H., Kusch, A., Gras, M., Cherel, Y., Maschette, D. \& Alderman, R. (2017) DNA metabarcoding as a marine conservation and management tool : A circumpolar examination of fishery discards in the diet of threatened Albatrosses. Frontiers in Marine Science, 4, 277. 
786 Meyer, J.M., Leempoel, K., Losapio, G. \& Hadly, E.A. (2020) Molecular ecological network 787 analyses : an effective conservation tool for the assessment of biodiversity, trophic interactions, and community structure. Frontiers in Ecology and Evolution, 8, 588430.

Nielsen, J.M., Clare, E.L., Hayden, B., Brett, M.T. \& Kratina, P. (2017) Diet tracing in ecology: method comparison and selection. Methods in Ecology and Evolution, 9, 278291.

Olaso, I., Sánchez, F., Rodríguez-cabello, C. \& Velasco, F. (2002) The feeding behaviour of some demersal fish species in response to artificial discarding. Scientia Marina, 66, 301311.

Palomares, M.L.D. \& Pauly, D. (2021) SeaLifeBase. World Wide Web electronic publication. www.sealifebase.org, version $(04 / 2021)$

Pinnegar, J.K. (2014) DAPSTOM - An Integrated Database \& Portal for Fish Stomach Records. Version 4.7. Centre for Environment, Fisheries \& Aquaculture Science, Lowestoft, UK.

Ramsay, K., Kaiser, M.J., Moore, P.G. \& Hughes, R.N. (1997) Consumption of fisheries discards by benthic scavengers: utilization of energy subsidies in different marine habitats. Journal of Animal Ecology, 66, 884-896.

Scheffer, M., Carpenter, S. \& de Young, B. (2005) Cascading effects of overfishing marine systems. Trends in Ecology \& Evolution, 20, 579-581.

Schnell, I.B., Bohmann, K. \& Gilbert, M.T.P. (2015) Tag jumps illuminated - reducing sequence-to-sample misidentifications in metabarcoding studies. Molecular Ecology Resources, 15, 1289-1303.

Sherley, R.B., Ladd-Jones, H., Garthe, S., Stevenson, O. \& Votier, S.C. (2019) Scavenger communities and fisheries waste : North Sea discards support 3 million seabirds, 2 million fewer than in 1990. Fish and Fisheries, 21, 132-145. 
811 da Silva, L.P., Mata, V.A., Lopes, P.B., Pereira, P., Jarman, S.N., Lopes, R.J. \& Beja, P.

812 (2019) Advancing the integration of multi-marker metabarcoding data in dietary analysis 813 of trophic generalists. Molecular Ecology Resources, 19, 1420-1432.

814 Taberlet, P., Bonin, A., Zinger, L. \& Coissac, E. (2018) Environmental DNA: For 815 Biodiversity Research and Monitoring. Oxford University Press, Oxford, UK.

816 Takahashi, M., DiBattista, J.D., Jarman, S., Newman, S.J., Wakefield, C.B., Harvey, E.S. \& 817 Bunce, M. (2020) Partitioning of diet between species and life history stages of 818 sympatric and cryptic snappers (Lutjanidae) based on DNA metabarcoding. Scientific $819 \quad$ Reports, 10, 4319.

820 Thompson, M.S.A., Pontalier, H., Spence, M.A., Pinnegar, J.K., Greenstreet, S., Moriarty, 821 M., Hélaouët, P. \& Lynam, C.P. (2020) A feeding guild indicator to assess 822 environmental change impacts on marine ecosystem structure and functioning. Journal 823 of Applied Ecology, 57, 1769-1781.

824 Tyson, C., Shamoun-baranes, J., Van Loon, E.E., Camphuysen, K.C.J. \& Hintzen, N.T. 825 (2015) Individual specialization on fishery discards by lesser black-backed gulls (Larus 826 fuscus). ICES Journal of Marine Science, 72, 1882-1891.

827 Votier, S.C., Bicknell, A., Cox, S.L., Scales, K.L. \& Patrick, S.C. (2013) A bird's eye view of 828 discard reforms : Bird-borne cameras reveal seabird/fishery interactions. PloS ONE, $\mathbf{8}$, $829 \quad$ e57376.

830 Zeller, D., Cashion, T., Palomares, M.-L. \& Pauly, D. (2018) Global marine fisheries 831 discards : A synthesis of reconstructed data. Fish and Fisheries, 19, 30-39. 